\title{
Recent Advances on Force Identification in Structural Dynamics
}

\author{
N. M. M. Maia, Y. E. Lage and M. M. Neves \\ Additional information is available at the end of the chapter \\ http://dx.doi.org/10.5772/51650
}

\section{Introduction}

This chapter presents recent advances on force identification for structural dynamics that have been developed by the authors using the concept of transmissibility for multiple degree-offreedom (MDOF) systems.

Being applied for many years only to the single degree-of-freedom (SDOF) system or to MDOF systems in a very limited way, the transmissibility concept has been developed along the last decade or so in a consistent manner, to be applicable in a general and complete way to MDOF systems. Various applications for MDOF systems may now be found, such as evaluation of unmeasured frequency response functions (FRFs), force identification, detection of damage, etc. A review of the multiple applications of the transmissibility concept has been published recently [1].

It is the application of this generalized transmissibility concept to both the direct and inverse force identification that is described along this chapter. The direct problem is understood as the one where one knows the applied forces and wishes to estimate the reactions at the supports; the inverse force identification problem is when one wishes to determine how many forces are applied, where they are applied and which are their magnitudes.

To determine the location and magnitude of the dynamic forces that excite the system is an important issue in structural dynamics [2,3], especially when operational forces cannot be directly measured, as it happens at inaccessible locations [4, 5]; it is often the case that transducers cannot be introduced in the structure to allow the experimental measurement of the external loads and only a limited number of sensors and positions are available. The identification of forces from vibration measurements at a few accessible locations is a 
very important problem in various areas, such as vibration control, fatigue life prediction and health monitoring.

Although the force identification problem may be solved from the dynamic responses by simply reversing the direct problem, this is usually ill-posed and sensitive to perturbations in the measured data.

Over the past years, the theory of inverse methods has been actively developed in many research areas presenting in common the effects of matrix ill-conditioning, reflecting the illposedness nature of the inverse problem itself. Those problems can often be overcome by methods such as pseudo-inversion for over-determined systems, use of Kalman filters [6, 7], Singular Value Decomposition and Tikhonov regularization [8-10].

Various research works in force identification can be found in the literature, such as those related to the identification of impact forces, implementation of prediction models based on reflected waves or simply from the dynamic responses [11-18], prediction of forces in plates for systems with time dependent properties [11] and identification of harmonic forces [13].

These methods to identify operational loads based on response measurements can be classified into three main categories: deterministic methods, stochastic methods and methods based on artificial intelligence. Two main classes of identification technique are considered in the group of deterministic methods for load identification: frequency-domain methods and time-domain methods. The force identification in time domain has been less studied than its frequency domain equivalent, therefore there are not that many force identification studies in the literature. A review on the state of the art for dynamic load identification may be found in [3, 14].

Although out of the scope of this chapter, some references are here given with respect to recent time-domain force identification developments. One interesting approach based on modal filtering [15] is the Sum of Weighted Accelerations Technique (SWAT), which allows to obtain the time-domain force reconstruction by isolating the rigid body modal accelerations. Another approach for time-domain force reconstruction is the Inverse Structural Filter (ISF) method of Kammer and Steltzner [16] that inverts the discrete-time equations of motion. A variant of this, expected to produce a stable ISF when the standard method fails was recently developed and named as Delayed Multi-step ISF (DMISF). For a more detailed description on these methods (SWAT, ISF and DMISF) see e.g. [17] and for its application to rotordynamics, see [18].

In this chapter, the authors treat the frequency-domain problem from a different perspective, which is based on the MDOF transmissibility concept. As aforementioned, usually the transmissibility of forces is defined in textbooks for SDOF systems, simply as the ratio between the modulus of the transmitted force magnitude to the support and the modulus of the applied force magnitude. For SDOF systems, the expression of either the transmissibility of motion or forces is exactly the same; however, as explained in [1], that is not the case for MDOF systems. On the one hand, the problem of extending the idea of transmissibility of motion to an MDOF system is essentially a problem of how to relate a set of unknown responses to a set of known responses associated to a given set of applied forces; on the other hand, for the transmissibility of forces the question is how to relate a set of reaction forces to a set of applied ones. 
Some initial attempts on the generalization of the transmissibility concept are due toVakakis et al. [19-21], Liu et al. [22, 23] and Varoto [24]. Similar efforts can also be found in the indirect measurement of vibration excitation forces $[2,4,5]$. To the best knowledge of the authors, a general answer to the problem is due to Ribeiro [25], and in [26] the experimental evaluation of the transmissibility concept for MDOF systems is presented. The concept of transmissibility of forces for MDOF has been proposed in 2006 [27], where the authors explain the formulation of the transmissibility using both the dynamic stiffness and the receptance matrices.

The use of the transmissibility in conjunction with a two step methodology for force identification is the main novelty of this chapter. For the force identification based on the transmissibility of motion, two steps are taken, (i) firstly the number of forces and their location are obtained, and (ii) secondly the reconstruction of the load vector is performed using some of the responses obtained experimentally together with the updated numerical model. Both have been numerically developed and implemented, as well as experimentally tested in the research group during the last years to access the potential of these new methods.

In section 2, the authors review the generalized transmissibility concepts, both in terms of displacements and forces. They are introduced and deduced from two different perspectives, (i) from the frequency response functions, (ii) from the dynamic stiffness.

In section 3, a numerical model and an experimental application are presented to illustrated the transmissibility concept.

In section 4 the methodologies proposed for force identification based on the transmissibility concept are introduced.

Some simulated and experimental results are presented to show how these methodologies are able to help us identifying applied and reaction forces. The authors present a discussion on these proposed methods and on the obtained results.

\section{Transmissibility in MDOF systems}

The transmissibility concept may be found in any fundamental textbook on mechanical vibrations (e.g. [28]), related to SDOF systems.

The transmissibility of motion is defined as the ratio between the modulus of the response amplitude (output) and the modulus of the imposed base harmonic displacement (input). Depending on the imposed frequency, the result can vary from an amplification to an attenuation in the response amplitude relatively to the input one.

On the other hand, the transmissibility of force is defined as the ratio between the modulus of the transmitted force magnitude to the support and the modulus of the imposed force magnitude.

It happens that for SDOF systems the expression for calculating the transmissibility is the same, either referring to forces or to motion. This is not the case for MDOF systems. 
The generalization of these definitions to MDOFs has been developed in the last decade, as mentioned before. In this section a brief review of these generalizations is given, introducing also the concepts and notation used for the force identification problem.

\subsection{Transmissibility of motion in MDOF systems}

To introduce the problem, the authors follow here as near as possible the notation used in [1]. Let $K$ be the set of $n_{K}$ co-ordinates where the displacement responses $Y_{K}$ are known (measured or computed), $U$ the set of $n_{U}$ co-ordinates where the displacement responses $Y_{U}$ are unknown, and $A$ the set of co-ordinates where the forces $\boldsymbol{F}_{A}$ may be applied (Fig. 1).

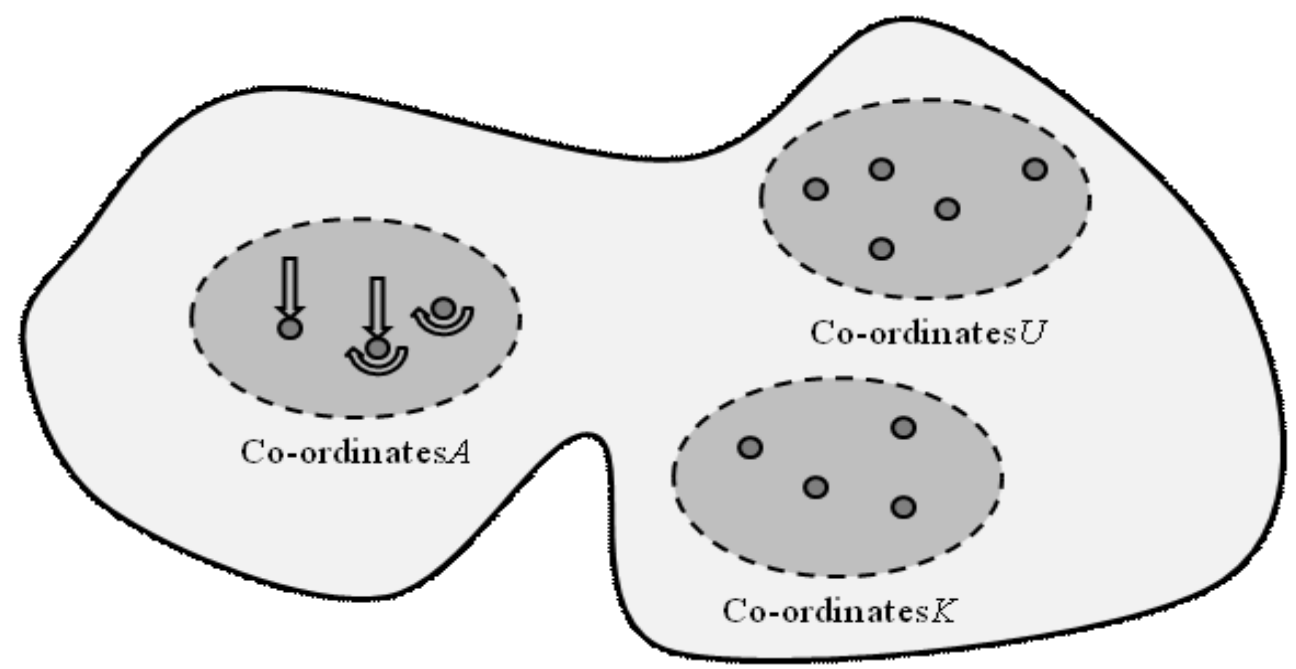

Figure 1. Illustration of an elastic body with the three sets of co-ordinates $K, U$ and $A$.

To obtain the needed transmissibility of motion one may consider two distinct ways. The first is based on the frequency response function (FRF) matrices $H(\omega)$, known as the fundamental formulation, while the second is based on the dynamic stiffness matrix $Z(\omega)$ and is named alternative formulation.

The receptance frequency response matrix $H(\omega)$ relates the dynamic displacement amplitudes $\boldsymbol{Y}$ with the external force amplitudes $\boldsymbol{F}$ as (using harmonic excitation, in steady-state conditions):

$$
\boldsymbol{Y}=\boldsymbol{H} \boldsymbol{F} \Leftrightarrow \boldsymbol{Y}=\left(\boldsymbol{K}-\omega^{2} \boldsymbol{M}+i \omega \boldsymbol{C}\right)^{-1} \boldsymbol{F}
$$

where $K, M$ and Care the stiffness, mass and viscous damping matrices, respectively. $H(\omega)$ includes all the degrees of freedom in which the system is discretized and corresponds to the 
inverse of the dynamic stiffness matrix $Z(\omega)$. One may underline that the mass-normalized orthogonality properties are observed here:

$$
\begin{aligned}
& \boldsymbol{\Phi}^{T} \boldsymbol{M} \boldsymbol{\Phi}=\mathbf{I} \\
& \boldsymbol{\Phi}^{T} K \boldsymbol{\Phi}=\operatorname{diag}\left(\omega_{r}^{2}\right)
\end{aligned}
$$

Assuming proportional damping, $C=\alpha K+\beta M$ and therefore,

$$
\boldsymbol{Y}=\boldsymbol{H} \boldsymbol{F}=\boldsymbol{\Phi}\left[\operatorname{diag}\left(\omega_{r}^{2}-\omega^{2}\right)+i \omega\left(\alpha \operatorname{diag}\left(\omega_{r}^{2}\right)+\beta \boldsymbol{I}\right)\right]^{-1} \boldsymbol{\Phi}^{T} \boldsymbol{F}
$$

where $\Phi$ is the mode shape matrix, $\omega_{r}$ is the $r^{\text {th }}$ natural frequency and $\alpha$ and $\beta$ are constants. From (1) it is easy to understand that if the responses $Y$ at the discretization points are known, then the force reconstruction (in frequency-domain) would be given by:

$$
\boldsymbol{F}=\boldsymbol{H}^{-1} \boldsymbol{Y}
$$

\subsubsection{Transmissibility of motion in terms of FRFs}

Based on harmonically applied forces at co-ordinates $A$, one may establish that displacements at co-ordinates $U$ and $K$ are related to the applied forces at co-ordinates $A$ by the following relationships:

$$
\begin{gathered}
\boldsymbol{Y}_{U}=\boldsymbol{H}_{U A} \boldsymbol{F}_{A} \\
\boldsymbol{Y}_{K}=\boldsymbol{H}_{K A} \boldsymbol{F}_{A}
\end{gathered}
$$

Eliminating the external forces $F_{A}$ between (5) and (6), one obtains

$$
\boldsymbol{Y}_{U}=\boldsymbol{H}_{U A}\left(\boldsymbol{H}_{K A}\right)^{+} \boldsymbol{Y}_{K}=\left(\boldsymbol{T}_{d}\right)_{U K}^{A} \boldsymbol{Y}_{K}
$$

where

$$
\left(\boldsymbol{T}_{d}\right)_{U K}^{A}=\boldsymbol{H}_{U A}\left(\boldsymbol{H}_{K A}\right)^{+}
$$

is the transmissibility matrix relating both sets of displacements. $\left(\boldsymbol{H}_{K A}\right)^{+}$is the pseudo-inverse of the sub-matrix $\boldsymbol{H}_{K A}$. An important property of the transmissibility matrix to be used here is that it does not depend on the magnitude of the involved forces and only requires the 
knowledge of a set of co-ordinates that include all the co-ordinates where the forces are applied. Indeed, it is required that $n_{K}$ be greater or equal to $n_{A}$. One important aspect of this definition is that sub-matrices $\boldsymbol{H}_{U A}$ and $\boldsymbol{H}_{K A}$ may be obtained experimentally.

\subsubsection{Transmissibility of motion in terms of dynamic stiffness}

There exists an alternative approach to obtain the transmissibility matrix for the displacements, using the dynamic stiffness matrices introduced in (1). Assuming again harmonic loading and defining two subsets, $A$ and $B, A$ being the set where the dynamic loads may be applied and $B$ the set formed by the remaining co-ordinates, where no forces are applied $\left(\boldsymbol{F}_{B}=\mathbf{0}\right)$, one can obtain (after grouping adequately the degrees of freedom of the problem):

$$
\left[\begin{array}{ll}
\boldsymbol{Z}_{A K} & \boldsymbol{Z}_{A U} \\
\boldsymbol{Z}_{B K} & \boldsymbol{Z}_{B U}
\end{array}\right]\left\{\begin{array}{l}
\boldsymbol{Y}_{K} \\
\boldsymbol{Y}_{U}
\end{array}\right\}=\left\{\begin{array}{c}
\boldsymbol{F}_{A} \\
\mathbf{0}
\end{array}\right\}
$$

Developing eq. (9), it follows that

$$
\begin{array}{ll}
\boldsymbol{Z}_{A K} Y_{K}+\boldsymbol{Z}_{A U} \boldsymbol{Y}_{U}=\boldsymbol{F}_{A} & \mathrm{a} \\
\boldsymbol{Z}_{B K} \boldsymbol{Y}_{K}+\boldsymbol{Z}_{B U} \boldsymbol{Y}_{U}=\mathbf{0} & \mathrm{b}
\end{array}
$$

From (10b) one obtains the transmissibility in terms of the dynamic stiffnesses:

$$
\boldsymbol{Y}_{U}=-\left(\boldsymbol{Z}_{B U}\right)^{+} \boldsymbol{Z}_{B K} \boldsymbol{Y}_{K}=\left(\boldsymbol{T}_{d}\right)_{U K}^{A} \boldsymbol{Y}_{K}
$$

where $\left(\boldsymbol{Z}_{B U}\right)^{+}$is the pseudo-inverse of $\boldsymbol{Z}_{B U}$.

From (11) it is possible to obtain the response at the unknown co-ordinates, as long as the pseudo-inverse is viable, which requires that $n_{B}$ is greater or equal to $n_{U}$.

Indeed, from all this resulted two conditions:

$$
\left(\boldsymbol{T}_{d}\right)_{U K}^{A}=-\left(\boldsymbol{Z}_{B U}\right)^{+} \boldsymbol{Z}_{B K}=\boldsymbol{H}_{U A}\left(\boldsymbol{H}_{K A}\right)^{+} \quad n_{B} \geq n_{U} \text { and } n_{K} \geq n_{A}
$$

\subsection{Transmissibility of forces in MDOF systems}

To introduce the transmissibility of forces for MDOF systems, the authors follow a similar procedure to the one used in the previous sub-section. The problem consists now of relating the set of known applied forces to a set of unknown reactions (or the other way around), relating the set of known applied forces (set $K$ ) with a set of unknown reaction forces (set $U$ ), which are illustrated in Fig.2. At the set $U$ it will be assumed that $\boldsymbol{Y}_{U}=\mathbf{0}$. In general, there will 
be other co-ordinates, where neither there are any applied forces nor there are any reactions, that shall constitute the set $C$.

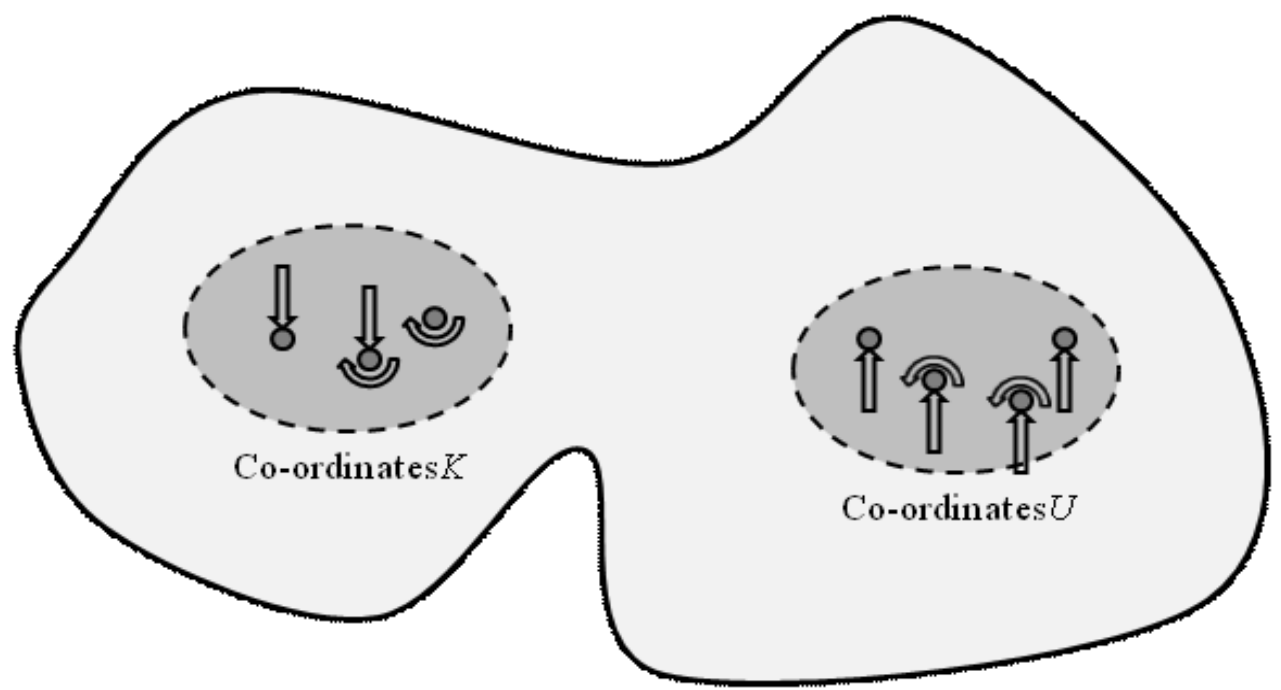

Figure 2. Illustration of both sets of co-ordinates $K$ and $U$.

\subsubsection{Transmissibility of forces in terms of FRFS}

With the definition of the new sets $K, U$ and $C$, the problem may be defined in the following way:

$$
\left\{\begin{array}{l}
\boldsymbol{Y}_{K} \\
\boldsymbol{Y}_{U} \\
\boldsymbol{Y}_{C}
\end{array}\right\}=\left[\begin{array}{ll}
\boldsymbol{H}_{K K} & \boldsymbol{H}_{K U} \\
\boldsymbol{H}_{U K} & \boldsymbol{H}_{U U} \\
\boldsymbol{H}_{C K} & \boldsymbol{H}_{C U}
\end{array}\right]\left\{\begin{array}{c}
\boldsymbol{F}_{K} \\
\boldsymbol{F}_{U}
\end{array}\right\}
$$

Imposing $Y_{U}=\mathbf{0}$, it follows that

$$
\boldsymbol{H}_{U K} \boldsymbol{F}_{K}+\boldsymbol{H}_{U U} \boldsymbol{F}_{U}=0
$$

and so

$$
\boldsymbol{F}_{U}=\left(\boldsymbol{T}_{f}\right)_{U K} \boldsymbol{F}_{K}
$$


where

$$
\left(\boldsymbol{T}_{f}\right)_{U K}=-\left(\boldsymbol{H}_{U U}\right)^{-1} \boldsymbol{H}_{U K}
$$

is the force transmissibility matrix.

This is the direct force identification method, i.e., one knows the applied forces and calculate the reactions at the supports, where the displacements are assumed as zero. The inverse problem is also possible, if one is able to measure the reaction forces and if their number is higher than the number of applied forces, in order to calculate the pseudo-inverse of $\boldsymbol{H}_{U K}$ :

$$
\boldsymbol{F}_{K}=\left(\left(\boldsymbol{T}_{f}\right)_{U K}\right)^{+} \boldsymbol{F}_{U}
$$

where

$$
\left(\left(\boldsymbol{T}_{f}\right)_{U K}\right)^{+}=-\left(\boldsymbol{H}_{U K}\right)^{+} \boldsymbol{H}_{U U}
$$

Note that in spite of the fact that here the reaction forces are known, the notation $U$ (that in principle stands for "unknown") is kept.

In the inverse problem, one may not know how many applied force exist and where they are applied. If that is the case, one must follow a different approach, as it will be explained in section 4.1

If the condition $Y_{U}=\mathbf{0}$ is relaxed, from eq. (13) it follows that:

$$
\begin{aligned}
& \qquad \boldsymbol{Y}_{U}=\boldsymbol{H}_{U K} \boldsymbol{F}_{K}+\boldsymbol{H}_{U U} \boldsymbol{F}_{U} \\
& \boldsymbol{F}_{U}=\left(\boldsymbol{T}_{f}\right)_{U K} \boldsymbol{F}_{K}+\left(\boldsymbol{H}_{U U}\right)^{-1} \boldsymbol{Y}_{U} \\
& \text { and } \\
& \boldsymbol{F}_{K}=\left(\left(\boldsymbol{T}_{f}\right)_{U K}\right)^{+} \boldsymbol{F}_{U}+\left(\boldsymbol{H}_{U K}\right)^{+} \boldsymbol{Y}_{U} \\
&
\end{aligned}
$$

\subsubsection{Transmissibility of forces in terms of dynamic stiffness}

Again, there is an alternative approach to obtain the force transmissibility matrix, using the dynamic stiffness matrices. 
Assuming harmonic loading and the mentioned sets $K, U$ and $C$, one can obtain (after grouping adequately the degrees of freedom of the problem) the following result:

$$
\left[\begin{array}{ccc}
\boldsymbol{Z}_{K K} & \boldsymbol{Z}_{K C} & \boldsymbol{Z}_{K U} \\
\boldsymbol{Z}_{C K} & \boldsymbol{Z}_{C C} & \boldsymbol{Z}_{C U} \\
\boldsymbol{Z}_{U K} & \boldsymbol{Z}_{U C} & \boldsymbol{Z}_{U U}
\end{array}\right]\left\{\begin{array}{l}
\boldsymbol{Y}_{K} \\
\boldsymbol{Y}_{C} \\
\boldsymbol{Y}_{U}
\end{array}\right\}=\left\{\begin{array}{c}
\boldsymbol{F}_{K} \\
\boldsymbol{F}_{C} \\
\boldsymbol{F}_{U}
\end{array}\right\}
$$

It is worthwhile noting that joining together the sets $K$ and $C$ in a new set $E$ makes it easier to see that imposing $\boldsymbol{Y}_{U}=\mathbf{0}$ one obtains the following relationships:

$$
\left[\begin{array}{cc}
\boldsymbol{Z}_{E E} & \boldsymbol{Z}_{E U} \\
\boldsymbol{Z}_{U E} & \boldsymbol{Z}_{U U}
\end{array}\right]\left\{\begin{array}{c}
\boldsymbol{Y}_{E} \\
\mathbf{0}
\end{array}\right\}=\left\{\begin{array}{c}
\boldsymbol{F}_{E} \\
\boldsymbol{F}_{U}
\end{array}\right\}
$$

from which it is clear that:

$$
\begin{array}{ll}
\boldsymbol{Z}_{E E} \boldsymbol{Y}_{E}=\boldsymbol{F}_{E} & \mathrm{a} \\
\boldsymbol{Z}_{U E} \boldsymbol{Y}_{E}=\boldsymbol{F}_{U} & \mathrm{~b}
\end{array}
$$

Eliminating $Y_{E}$ between (23a) and (23b), it turns out that

$$
\boldsymbol{F}_{U}=\left(\boldsymbol{T}_{f}\right)_{U E} \boldsymbol{F}_{E}
$$

where

$$
\left(\boldsymbol{T}_{f}\right)_{U E}=\boldsymbol{Z}_{U E}\left(\boldsymbol{Z}_{E E}\right)^{-1}
$$

The inverse problem corresponds to

$$
\boldsymbol{F}_{E}=\left(\left(\boldsymbol{T}_{f}\right)_{U E}\right)^{+} \boldsymbol{F}_{U}
$$

with

$$
\left(\left(\boldsymbol{T}_{f}\right)_{U E}\right)^{+}=\boldsymbol{Z}_{E E}\left(\boldsymbol{Z}_{U E}\right)^{+}
$$


It is important to note that only some of the co-ordinates of the set $E$ have applied forces. This means that in (23) some rows of $\boldsymbol{F}_{E}$ are zero and only the columns (in $\boldsymbol{Z}_{E E}$ ) whose co-ordinates have applied forces (set $K$ ) are needed for the transmissibility matrix. In other words, from the set $E$ only the co-ordinates corresponding to the $K$ set are used.

\subsection{Summary}

From sections 2.2.1 and 2.2.2, one can conclude that for the direct problem of transmissibility of forces there is no restrictions in the number of co-ordinates used:

$$
\begin{aligned}
& \left(\boldsymbol{T}_{f}\right)_{U K}=-\left(\boldsymbol{H}_{U U}\right)^{-1} \boldsymbol{H}_{U K} \\
& \left(\boldsymbol{T}_{f}\right)_{U E}=\boldsymbol{Z}_{U E}\left(\boldsymbol{Z}_{E E}\right)^{-1}
\end{aligned}
$$

whereas in the inverse problem of transmissibility of forces there are some restrictions that can make this option not very useful in practice, especially when using the dynamic stiffnesses, since one needs to calculate the pseudo-inverse matrices:

$$
\begin{array}{ll}
\left(\left(\boldsymbol{T}_{f}\right)_{U K}\right)^{+}=-\left(\boldsymbol{H}_{U K}\right)^{+} \boldsymbol{H}_{U U} & n_{U} \geq n_{K} \\
\left(\left(\boldsymbol{T}_{f}\right)_{U E}\right)^{+}=\boldsymbol{Z}_{E E}\left(\boldsymbol{Z}_{U E}\right)^{+} & n_{U} \geq n_{E}
\end{array}
$$

\section{Numerical and experimental applications}

As explained before, the transmissibility matrices may be obtained from a numerical model (which should be updated for the range of frequencies involved) or from results obtained experimentally. In this section, the methodology used in each case is described and illustrated through a comparison example.

\subsection{Transmissibility in terms of the numerical model}

For the numerical model, one needs the knowledge of the structure within the discretization chosen, to create the receptance matrix $H(\omega)$, which is the inverse of the corresponding dynamic stiffness matrix $Z(\omega)$. Here, the numerical model is created using the Finite Element Method (FEM), although other alternatives may also be used. As seen before, the dynamic stiffness matrix is defined as:

$$
\boldsymbol{Z}(\omega)=\boldsymbol{K}-\omega^{2} \boldsymbol{M}+i \omega \boldsymbol{C}
$$


where $C$ represents the viscous damping matrix, often of the proportional type, i.e., $C=\alpha K+\beta M$, where $\alpha$ and $\beta$ are constants to be evaluated experimentally.

To build the dynamic stiffness matrix, a specific structural finite element is chosen according to the approximation considered. For example, in the case of a reasonably long and slender beam one can use the Euler-Bernoulli beam element (instead of a shell or solid structural element). Then, the global matrices are assembled for the chosen discretization of the structure.

In order to improve the accuracy of the numerical model when simulating what is obtained experimentally, concentrated masses are often added at the corresponding nodes to model the effect of the accelerometers used in the testing positions.

Although the receptance matrix $H(\omega)$ is the inverse of the corresponding dynamic stiffness matrix, one should avoid such direct numerical inversion (frequency by frequency). Instead, $H(\omega)$ is calculated from eq. (3), after a modal analysis in free vibration.

Then, using (8) or (16), one can calculate the needed transmissibility matrices $\left(\boldsymbol{T}_{d}\right)_{U K}{ }^{A}$ or $\left(\boldsymbol{T}_{f}\right)_{U K}$. Of course, alternatively one may use the equivalent expressions (11) or (25), respectively.

\subsection{Transmissibility in terms of experimental measurements}

Depending on the type of transmissibility to obtain, the corresponding experimental setup should be established. Essentially, it is important to observe that for the transmissibility of motion one measures the FRFs relating co-ordinates $U$ and $K$ with co-ordinates A, normally using accelerometers and force transducers. For validation purposes, one may also measure the applied forces. In the examples presented next for the transmissibility of motion, a suspended (free-free) beam is always used.

For the transmissibility of forces, in the direct problem, one measures the applied forces at coordinates $K$ (in the inverse problem, one measures the reaction forces at co-ordinates $U$ ). For validation purposes, one also measures the ones to be estimated. The test specimen for the transmissibility of forces is always a simply supported beam.

For the experimental setup, the following equipment is used:

- Vibration exciter (Brüel \& Kjær Type 4809);

- Power amplifier (Brüel \& Kjær Type 2706);

- Force transducers(PCB PIEZOTRONICS Model 208C01);

- Data acquisition equipment (Brüel \& Kjær Type 3560-C).

In Fig. 3, a schematic representation of the experimental setup used for the force transmissibility tests is presented, in this case a simply supported beam with a single applied force.

The excitation signal used was a multi-sine transmitted to the exciter, with constant amplitude in the frequency. In reality the signal measured by the force transducer does not exhibits a constant amplitude along the frequency, as it depends on the dynamic response of the structure. 


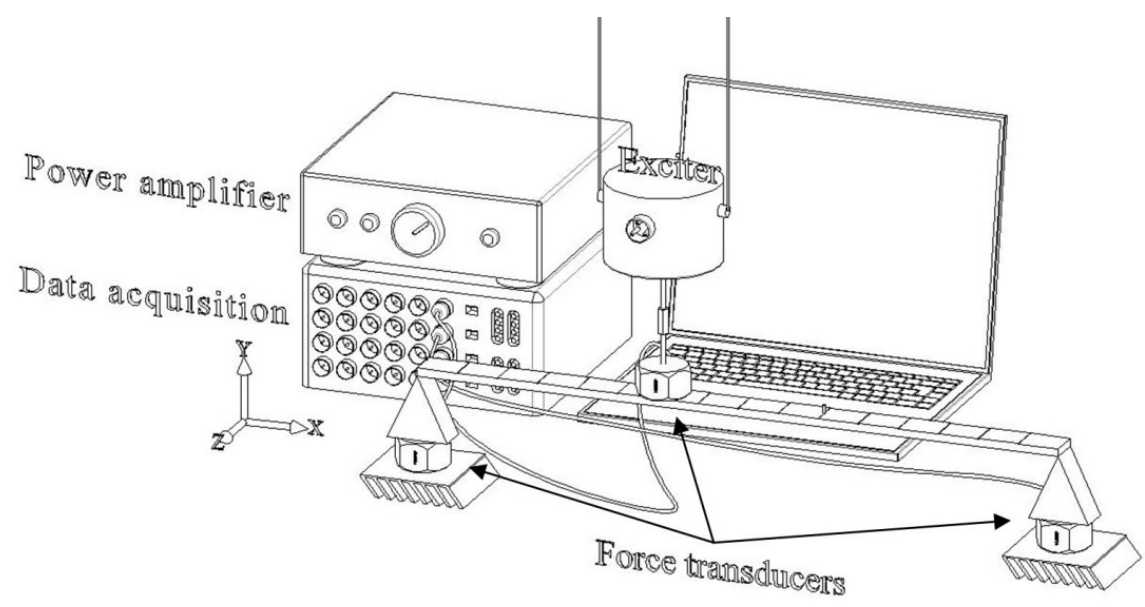

Figure 3. Example of the experimental setup developed for the transmissibility of forces.

For the transmissibility of motion, a beam suspended by nylon strings is used, to simulate freefree conditions. In order to facilitate the interchange of the available accelerometers between the measure positions without affecting the dynamics of the structure, it is important to add equivalent masses (dummies) to model the effect of the sensors.

After obtaining experimentally the needed receptances, by using (8) or (16) one can establish the transmissibility matrices $\left(\boldsymbol{T}_{d}\right)_{U K}{ }^{A}$ or $\left(\boldsymbol{T}_{f}\right)_{U K}$.

\subsection{Examples}

The same steel beam was used in all the examples. With the purpose of illustrating the applicability of the presented formulations to obtain the transmissibility plots, the authors used the geometric and material parameters presented in Table 1. Note that these data correspond to the values obtained after updating the FE model.

\begin{tabular}{lc}
\hline Young's modulus - E & $208 \mathrm{GPa}$ \\
\hline Density - $\rho$ & $7840 \mathrm{~kg} / \mathrm{m}^{3}$ \\
\hline Length - L & $0.8 \mathrm{~m}$ \\
\hline Section width - b & $5.0 \times 10^{-3} \mathrm{~m}$ \\
\hline Section height - $\mathrm{h}$ & $20.0 \times 10^{-3} \mathrm{~m}$ \\
\hline Section area - A & $1 \times 10^{-4} \mathrm{~m}^{2}$ \\
\hline Second moment of area - I & $2.0883 \times 10^{-10 \mathrm{~m}^{4}}$ \\
\hline proportional damping - $a$ & $4 \mathrm{~s}$ \\
\hline proportional damping - $\beta$ & $2.0 \times 10^{-6} \mathrm{~s}^{-1}$
\end{tabular}

Table 1. Beam properties (after updating). 


\subsubsection{Numerical model}

The standard two-node Euler-Bernouli bidimensional finite element is used here to build the needed numerical model of the beam.

The beam was discretized into sixteen finite elements, which correspond to $N=17$ nodes, ordered from $1 \mathrm{up}$ to 17 . As the analysis and the model are limited to the plane $\mathrm{xOy}$, each node has three degrees of freedom (which are $u_{x^{\prime}} u_{y^{\prime}} \theta$ ). Hence, the matrices of the numerical model have an order of $3 x \mathrm{~N}$ for the free-free beam. In what the measurements are concerned, only the displacements and applied forces along the $y$ direction are used and therefore the numbering of nodes and co-ordinates $y$ coincide.

The model was updated using $E, \rho$ and $I$ as updating parameters and a proportional damping model is included using $\alpha$ and $\beta$ as updating parameters (Table 1).

\subsubsection{Example 1 - Transmissibility of motion}

In this example, the free-free beam has only one applied force at node 11 along the $y$ direction, denoted as $F_{11}$, and the measurements are taken at nodes 3, 7, 13 and 17 also along the $y$ direction, denoted as $\mathrm{Y}_{3}, \mathrm{Y}_{7}, \mathrm{Y}_{13}$, and $\mathrm{Y}_{17}$ (Fig. 4).

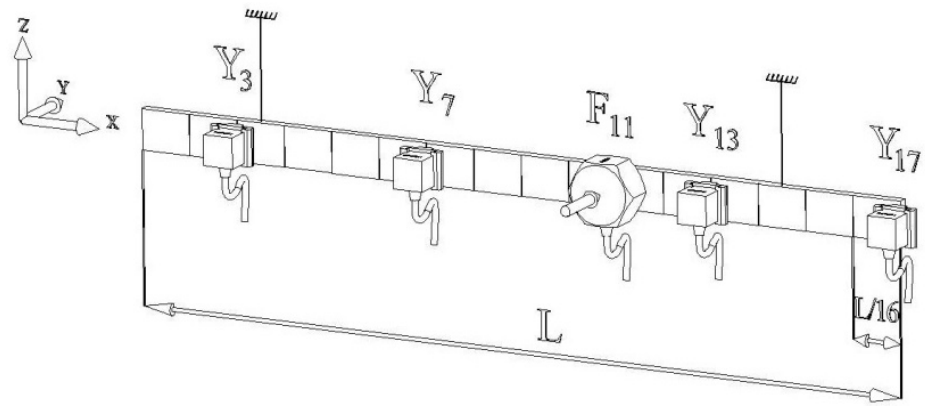

Figure 4. Schematic representation of the accelerometers and force transducer positions.

The transmissibility of motion between the pair of nodes $(3,7)$ and the pair of nodes $(13,17)$ may be expressed as

$$
\boldsymbol{Y}_{U}=\left(\mathbf{T}_{d}\right)_{U K}^{A} \boldsymbol{Y}_{K} \Leftrightarrow\left\{\begin{array}{l}
Y_{13} \\
Y_{17}
\end{array}\right\}=\left[\begin{array}{ll}
T_{13,3} & T_{13,7} \\
T_{17,3} & T_{17,7}
\end{array}\right]\left\{\begin{array}{c}
Y_{3} \\
Y_{7}
\end{array}\right\}
$$

These transmissibilities were obtained from eq. (8), using the FRFs calculated from the numerical model and measured experimentally. Two of them are plotted in Fig. 5, where one can observe that both ways are able to produce the transmissibility response of the structure, as they match reasonably well. 

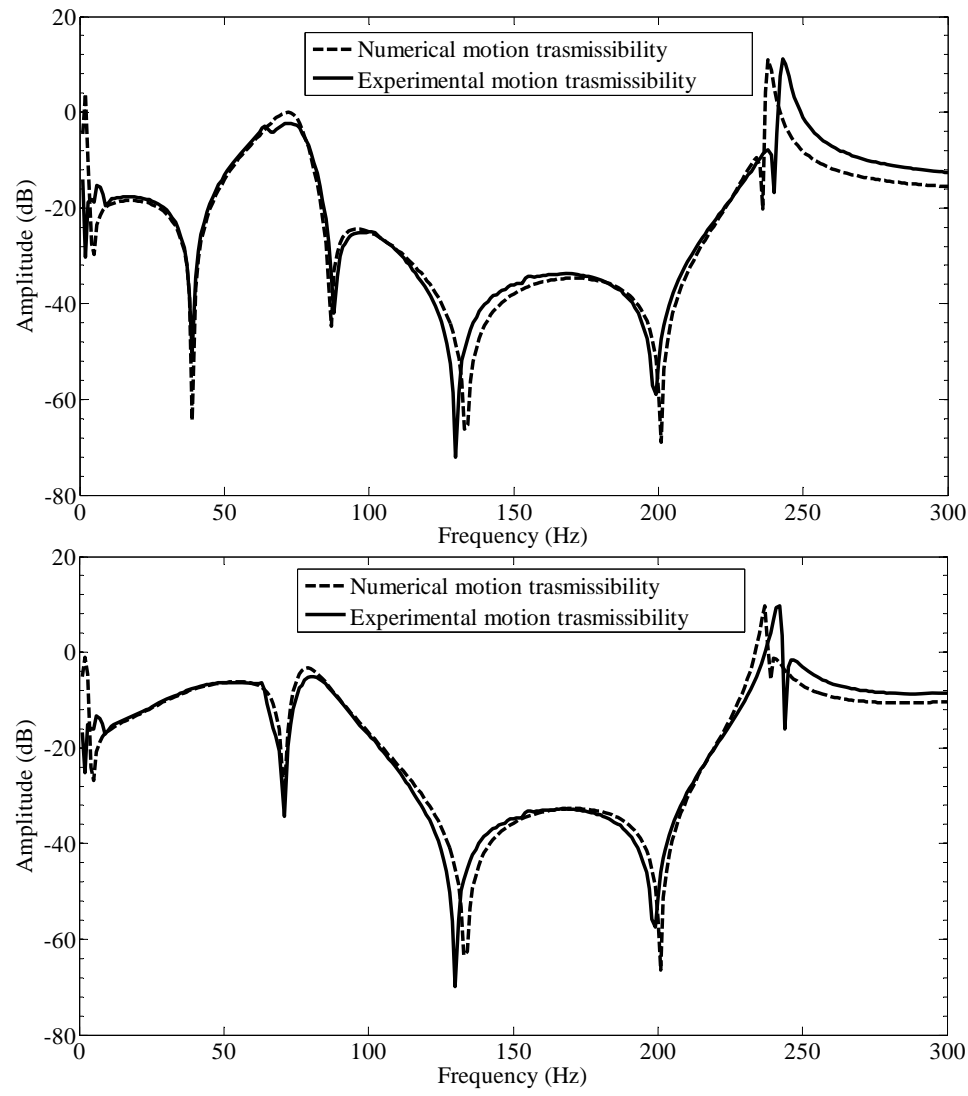

Figure 5. Numerical and experimental transmissibilities (upper plot, $T_{13,3} ;$ bottom plot, $T_{13,7}$ )

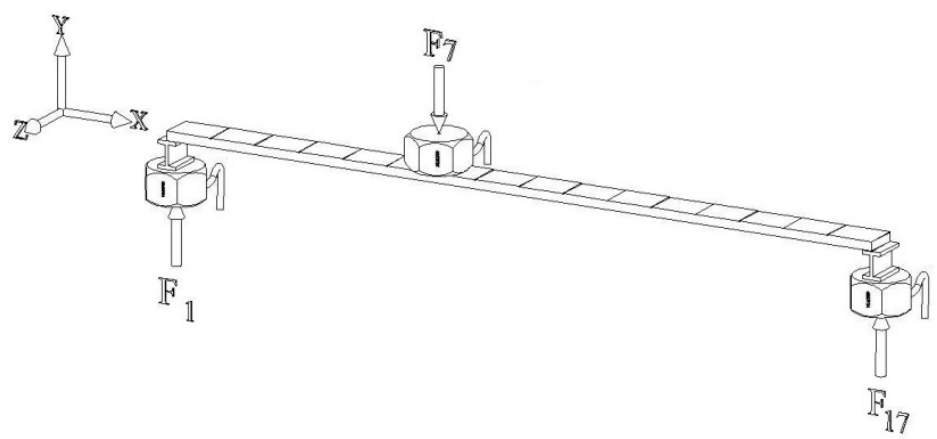

Figure 6. Schematic representation of the positions of the force transducers. 


\subsubsection{Example 2 - Transmissibility of forces}

In this case, a simply supported beam is considered with one applied force at node 7 and reactions at nodes 1 and 17. Only the magnitude of the forces is measured, and the transmissibility is obtained directly from the measurements and compared with the numerical results. The experimental setup is illustrated in Fig. 6.

The force transmissibility relation between node 7 and the pair of nodes $(1,17)$ may be expressed as

$$
\left\{\begin{array}{c}
F_{1} \\
F_{17}
\end{array}\right\}=\left[\begin{array}{c}
T_{1,7} \\
T_{17,7}
\end{array}\right]\left\{F_{7}\right\}
$$
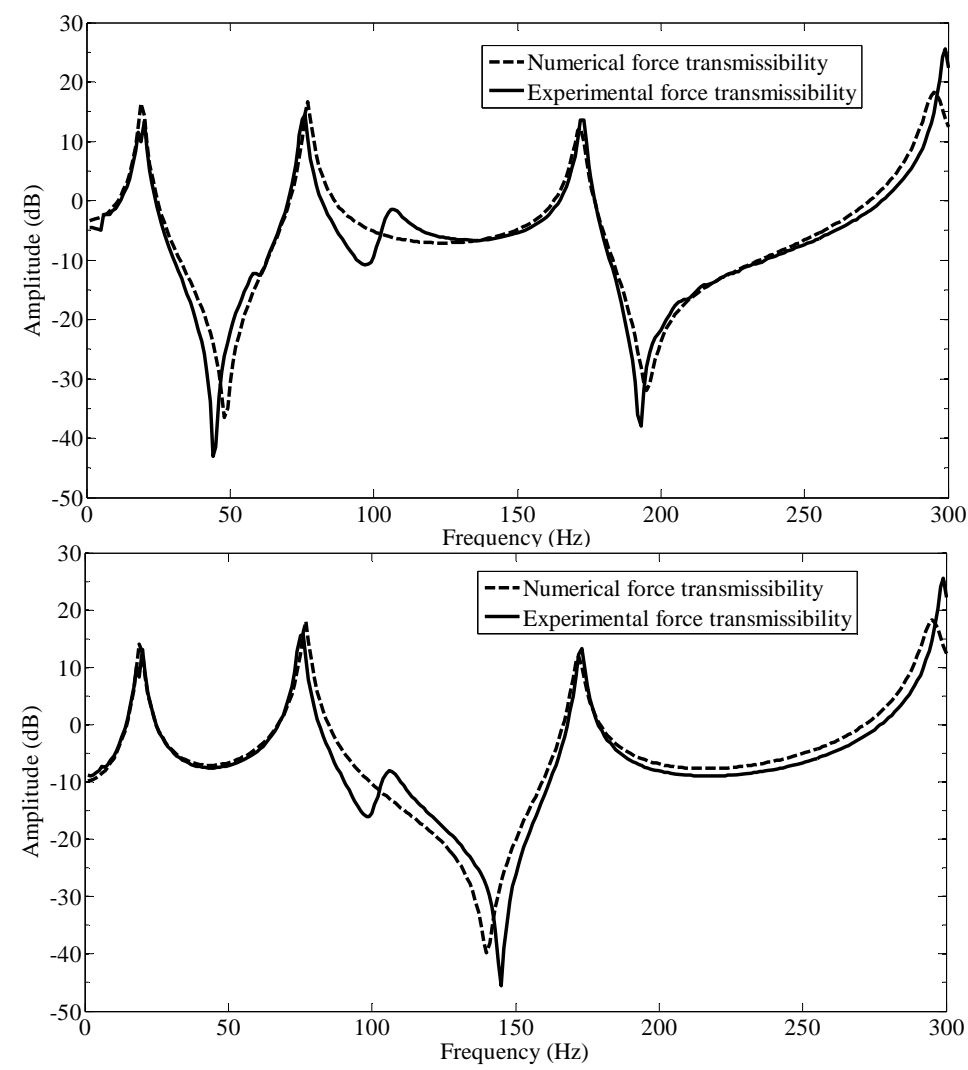

Figure 7. Numerical and experimental transmissibilities (upper plot, $T_{1,7}$; bottom plot, $T_{17,7}$ ) 
The force transmissibilities were obtained using eq. (16) and are plotted in Fig. 7, where it is clear that both numerical and experimental FRFs are able to produce the transmissibility response of the structure. Note that around $100 \mathrm{~Hz}$ there is a "bump" in the experimental curve, due to the effect of the supports of the beam themselves; this effect has not been included in the numerical model because it was not important, as these results are only of an illustrative type.

\section{Force identification}

This section shall be divided into (i) part one for the force localization algorithm based on the transmissibility of motion and reconstruction using the measured responses and the updated numerical model, and (ii) part two for the force reconstruction using the transmissibility of forces.

\subsection{Force localization based on the transmissibility of motion and force reconstruction}

The force identification problem is a difficult matter, as one has a limited knowledge of the measured responses, due to the complexity of the structure, lack of access to some locations, etc. In other words, there are difficulties due to the incompleteness of the model.

Due to this difficulty in calculating the load vector directly, the authors propose to divide the process into two distinct steps:

1. the localization of the forces, i.e. the identification of the number and position of the applied forces using the concept of transmissibility of motion;

2. the load vector reconstruction.

For the first step, a search for the number and position of forces using the transmissibility of motion is performed. Essentially, this step consists of searching for the transmissibility matrix correspondent to the dynamics of the system and using the available measured data and the numerical model involved.

Once the corresponding transmissibility matrix is found, one has a solution for the number and position of the forces applied to the structure.

The second step consists of reconstructing the load vector with the results obtained in the first step. A more detailed description about this methodology is given in the following sections.

\subsubsection{Force localization}

In a first stage, to apply the method proposed in the previous section, one finds the transmissibility matrix that converts the dynamic responses $Y_{K}$ into $Y_{U}$. As one does not know the position of the applied forces, it was decided to cover all the possibilities until the calculated responses $\left(\boldsymbol{Y}_{U}\right)$ match the measured ones $\tilde{\boldsymbol{Y}}$, over a range of frequencies. To calculate the vector $Y_{U}$ one may use either eq. (7) or (11). 
The maximum number of forces must be less or equal to the dimension of the known dynamic response vector $\boldsymbol{Y}$.

The successive combinations of the tested nodes are obtained according to the following scheme:

For one force: $\quad\{(1), \ldots(\mathrm{N})$;

For two forces : $\quad\left\{\begin{array}{cc}(1,2), \ldots & (1, \mathrm{~N}) \\ (2,3), \ldots & (2, \mathrm{~N}) \\ (3,4), \ldots & (3, \mathrm{~N}) \\ \vdots & \\ (N-1, N) & \end{array}\right.$

For three forces : $\left\{\begin{array}{c}(1,2,3), \ldots(1,2, N) \\ \vdots\end{array}\right.$

The error in each combination is kept in a vector to identify the combination with the least associated error (in absolute value). Firstly, the algorithm scrolls through the possible combinations of position and number of forces. For each combination, the associated error between the calculated vector $Y_{U}$ and the measured response vector $\tilde{\boldsymbol{Y}}$ is calculated; this is carried out over a frequency range defined by the user. The error between the predicted and the measured dynamic response at each co-ordinate $i$ can be defined as:

$$
\operatorname{error}_{i}=\sum_{\omega}\left(\log \left(\operatorname{abs}\left(\tilde{Y}_{U_{i}}(\omega)\right)\right)-\log \left(\operatorname{abs}\left(Y_{U_{i}}(\omega)\right)\right)\right)^{2}
$$

For each combination, the calculated error is kept in an entry of the error vector and analyzed later on:

$$
\boldsymbol{\varepsilon}=\left\{\text { error }_{i}\right\}
$$

The accumulated error for a given combination of co-ordinates where $\boldsymbol{F}$ can be located is the norm of $\varepsilon$. The calculations are repeated for sucessive combinations of number and position of forces. The combination of the force locations that gives the lowest error leads to the number and position of the forces applied to the structure. As already mentioned, the maximum number of forces that can be found is equal to the dimension of the known dynamic response vector.

As one does not know a priori how many forces exist, one has to follow a trial and error procedure that consists basically in assuming an increasing number of forces and the corresponding number of measurements; if the right number of forces is $N_{f}$, one has a minimum 
error $\varepsilon$ for a certain set of co-ordinates. When one proceeds and assumes $N_{f}+1$ forces and measurements, the error will be higher then $\varepsilon$, telling us that the right answer was effectively $N_{f}$ at a certain set of co-ordinates.

It is clear that all the combinations of the $N_{f}+1$ forces that contain the right combination of the $N_{f}$ forces should exhibit a local minimum, though not the absolute one.

The method was implemented computationally (in MatLab ${ }^{\circledR}$ ).

\subsubsection{Force reconstruction}

In a second step, the reconstruction of the force amplitudes consists of solving an inverse problem using the measured dynamic responses $Y_{\mathrm{K}}$ :

$$
\boldsymbol{F}_{A}=\left(\boldsymbol{H}_{K A}\right)^{+} \boldsymbol{Y}_{K}
$$

Note that for the given system to be invertible, the number of dynamic responses to be used (set K) must be higher or equal than the number of applied forces (set A). However, this is always verified, as in the first step one has already imposed it.

\subsubsection{Example 3 - Localization of the applied forces}

This is a numerical example, illustrated in Fig.8, where a set of uncorrelated forces is applied at co-ordinates 1 and 5 (set $A$ ), and one uses the three known responses (set $K$ ) to identify the number and location of forces.

A set of simulated results (to mimic the experimental measurements) are obtained at nodes 1 , 3, 5, 11 and 17 (see Fig. 8); they define the following sets:

$$
\mathbf{Y}_{K}=\left\{\begin{array}{lll}
Y_{3} & Y_{5} & Y_{17}
\end{array}\right\}^{T} \text { and } \mathbf{Y}_{U}=\left\{\begin{array}{ll}
Y_{1} & Y_{11}
\end{array}\right\}^{T}
$$

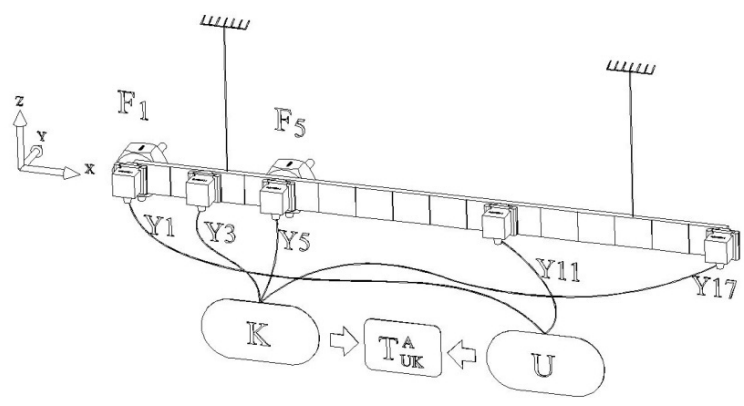

Figure 8. Illustration with the responses and applied force locations for example 3. 
Considering these responses, the maximum number of identifiable applied forces is three, as explained before. The forces are uncorrelated and applied to the structure at co-ordinates 1 and 5. A series of force combinations have to be systematically generated as follows. In this case, all combinations up to three forces have been considered:

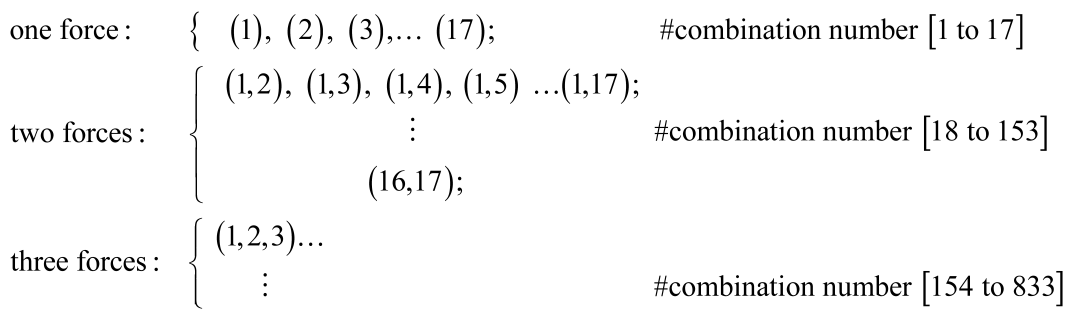

Applying the localization method described in the previous subsection 4.1.1, one obtains the plot of the error defined in eq. (33), as shown in Fig.9.

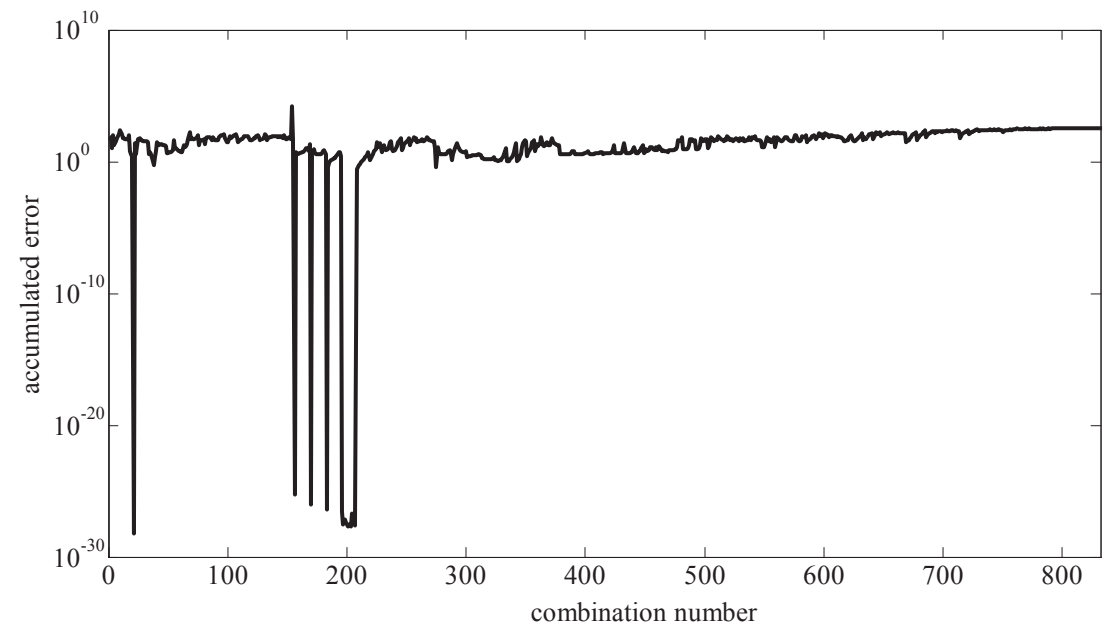

Figure 9. Accumulated error in frequency for each combination of forces.

It is clear that there are several situations (combinations) where the error is close to zero and other where is not.

The minimum error happens with the combination number 21, corresponding to two forces applied at co-ordinates 1 and 5, thus identifying the correct positions and number of forces (Table 2). 


\begin{tabular}{cccccc}
\hline Combination & Number of forces & Position of the forces & $\begin{array}{c}\text { Number of } \\
\text { identified forces }\end{array}$ & $\begin{array}{c}\text { Identified } \\
\text { positions }\end{array}$ & $\begin{array}{c}\text { Absolute } \\
\text { error }\end{array}$ \\
\hline 21 & 2 & 1,5 & 2 & 1,5 & $2,69 \mathrm{e}-29$ \\
\hline
\end{tabular}

Table 2. Data of the combination with minimum error.

To better understand why there exist more combinations with small errors, Table 3 shows these combinations with its corresponding error value. All of them have a common group of coordinates, corresponding to the correct combination of number and positions of the forces. In this case the correct positions are obtained with success through the minimum error.

\begin{tabular}{ccc}
\hline Combination & Real position of the forces & Absolute error \\
\hline 21 & 1,5 & $2,69 \mathrm{e}-29$ \\
\hline 156 & $1,2,5$ & $7,99 \mathrm{e}-26$ \\
\hline 170 & $1,3,5$ & $2,85 \mathrm{e}-27$ \\
\hline 183 & $1,4,5$ & $6,99 \mathrm{e}-28$ \\
\hline 197 & $1,5,7$ & $8,70 \mathrm{e}-29$ \\
\hline 198 & $1,5,8$ & $1,86 \mathrm{e}-28$ \\
\hline 199 & $1,5,9$ & $1,82 \mathrm{e}-28$ \\
\hline 200 & $1,5,10$ & $5,54 \mathrm{e}-28$ \\
\hline
\end{tabular}

Table 3. Some combinations and their respective error.

This illustrates the localization step performed with two forces, whose number and location were not known at the beginning. From these results, it can be stated that the transmissibility of motion can be considered as adequate to perform this task. Note that, in spite of the high numbers of combinations that exist, the computations are relatively quick, as they involve only sub-matrices, and for the first permutations they are of a small order.

\subsubsection{Example 4 - Localization and reconstruction 1}

This is an experimental example, where a multisine signal is fed into the shaker, attached to the beam at co-ordinate 13. Later on, the applied force is compared with the reconstructed one. The experimental measurements are obtained at nodes 5, 7, 11 and 15. The measured vectors are as follows:

$$
\mathbf{Y}_{K}=\left\{\begin{array}{ll}
Y_{7} & Y_{15}
\end{array}\right\}^{T} \text { and } \mathbf{Y}_{U}=\left\{\begin{array}{ll}
Y_{5} & Y_{11}
\end{array}\right\}^{T}
$$


Considering these responses, the maximum number of identifiable applied forces is two, as explained before.

A series of force combinations was systematically generated as described before. Applying the localization method, one obtains the graph of Fig. 10.

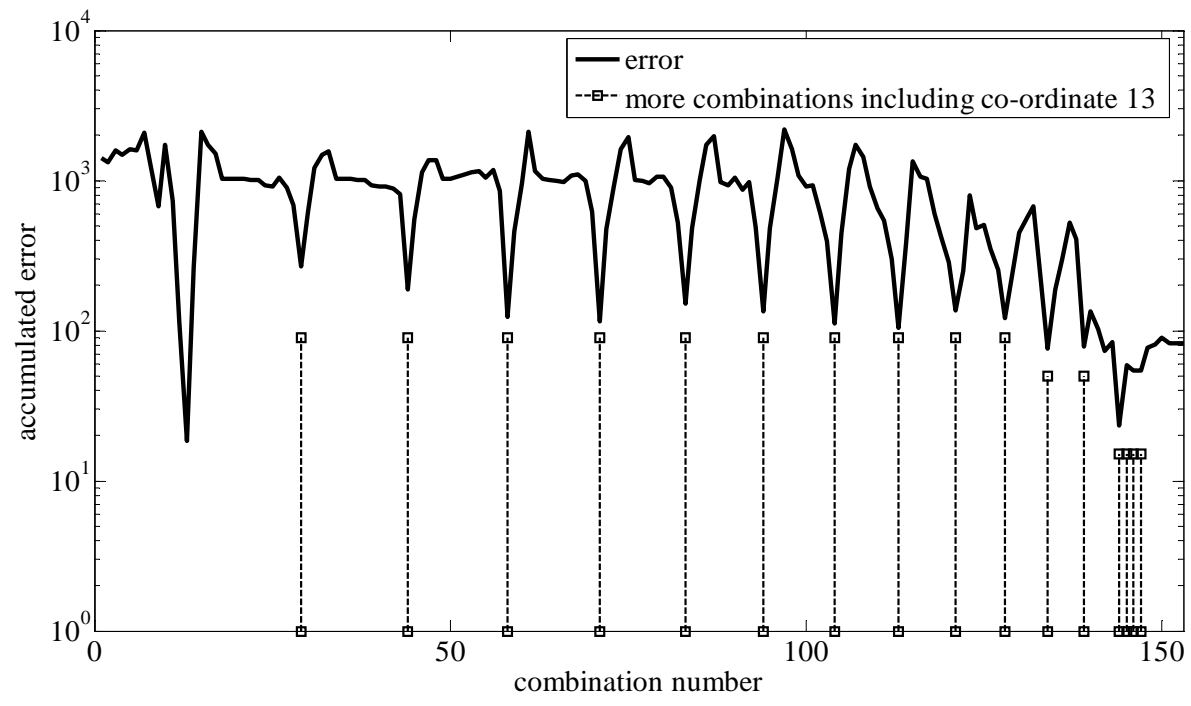

Figure 10. Accumulated error in frequency for each force combination.

As one can see, the absolute minimum corresponds to combination no. 13, which is right because this combination represents the force applied at co-ordinate 13. So, the method could localize correctly the position of the force at co-ordinate 13.

Once the localization of the force is accomplished, its reconstruction is a simple calculation, using the measured displacements relating those co-ordinates to the force location. As the force is located at node 13, taking the measurement at co-ordinates 5, 7, 11 and 15, for instance, it follows that:

$$
\left\{\begin{array}{c}
\tilde{Y}_{5} \\
\tilde{Y}_{7} \\
\tilde{Y}_{11} \\
\tilde{Y}_{15}
\end{array}\right\}=\left\{\begin{array}{l}
H_{5,13} \\
H_{7,13} \\
H_{11,13} \\
H_{15,13}
\end{array}\right\} F_{13} \Leftrightarrow F_{13}=\left\{\begin{array}{c}
H_{5,13} \\
H_{7,13} \\
H_{11,13} \\
H_{15,13}
\end{array}\right\}+\left\{\begin{array}{c}
\tilde{Y}_{5} \\
\tilde{Y}_{7} \\
\tilde{Y}_{11} \\
\tilde{Y}_{15}
\end{array}\right\}
$$


Using the information from the measured responses, the reconstruction is now immediate. To validate this methodology, the result was ploted against its experimentally measured curve, as in Fig. 11. One may affirm that the method is able to predict the applied force. It is possible that a better matching of the curves may be obtained with a finer updated FE model.

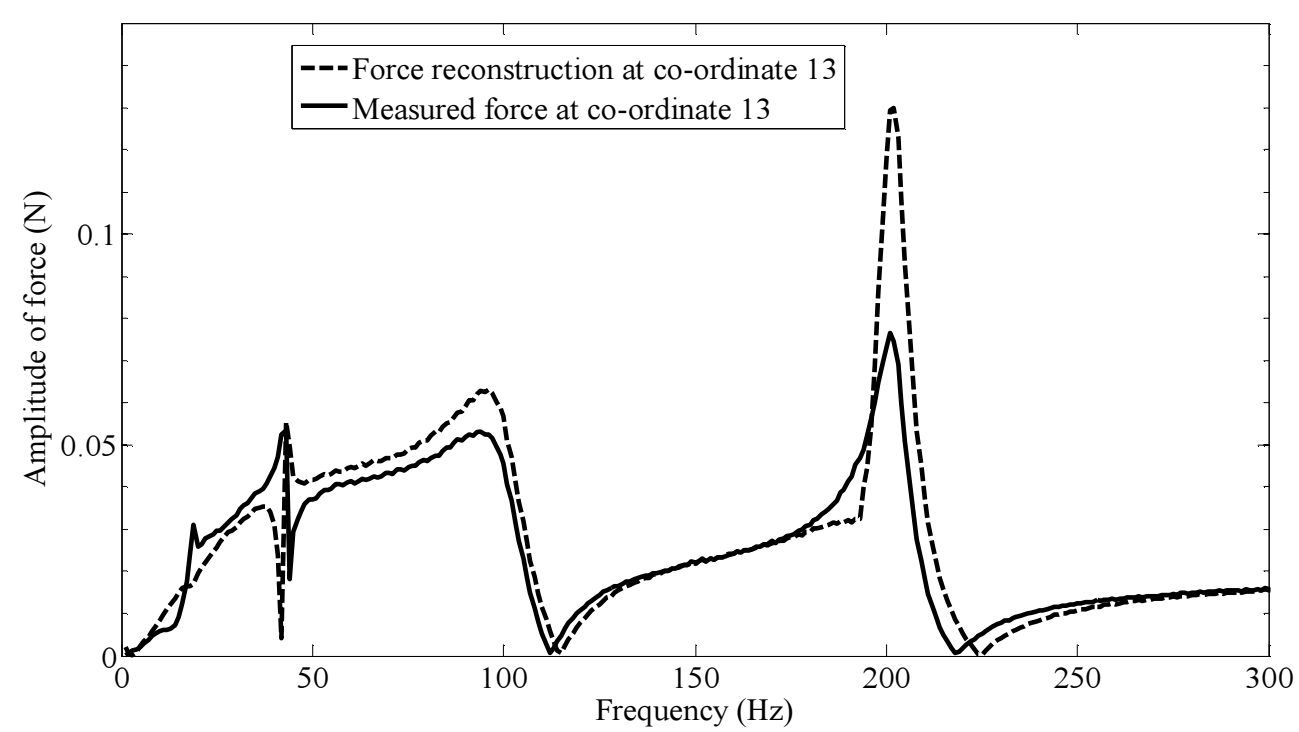

Figure 11. Comparison between the experimental and the reconstructed forces at co-ordinate 13.

\subsubsection{Example 5 - Localization and reconstruction 2}

Here, the same multisine signal is fed into two shakers, attached to the free-free beam at the co-ordinates 1 and 11. Later on, the applied forces are compared with the reconstructed ones. The experimental measures are obtained at nodes $3,7,17$ and 13 . The measured vectors are as follows.

$$
\mathbf{Y}_{K}=\left\{Y_{3}, Y_{7}, Y_{17}\right\}^{T} \text { and } \quad \mathbf{Y}_{U}=\left\{Y_{13}\right\}
$$

Considering these responses, the maximum number of identifiable applied forces is three. Applying the localization method, one obtains the plot shown in Fig.12. 


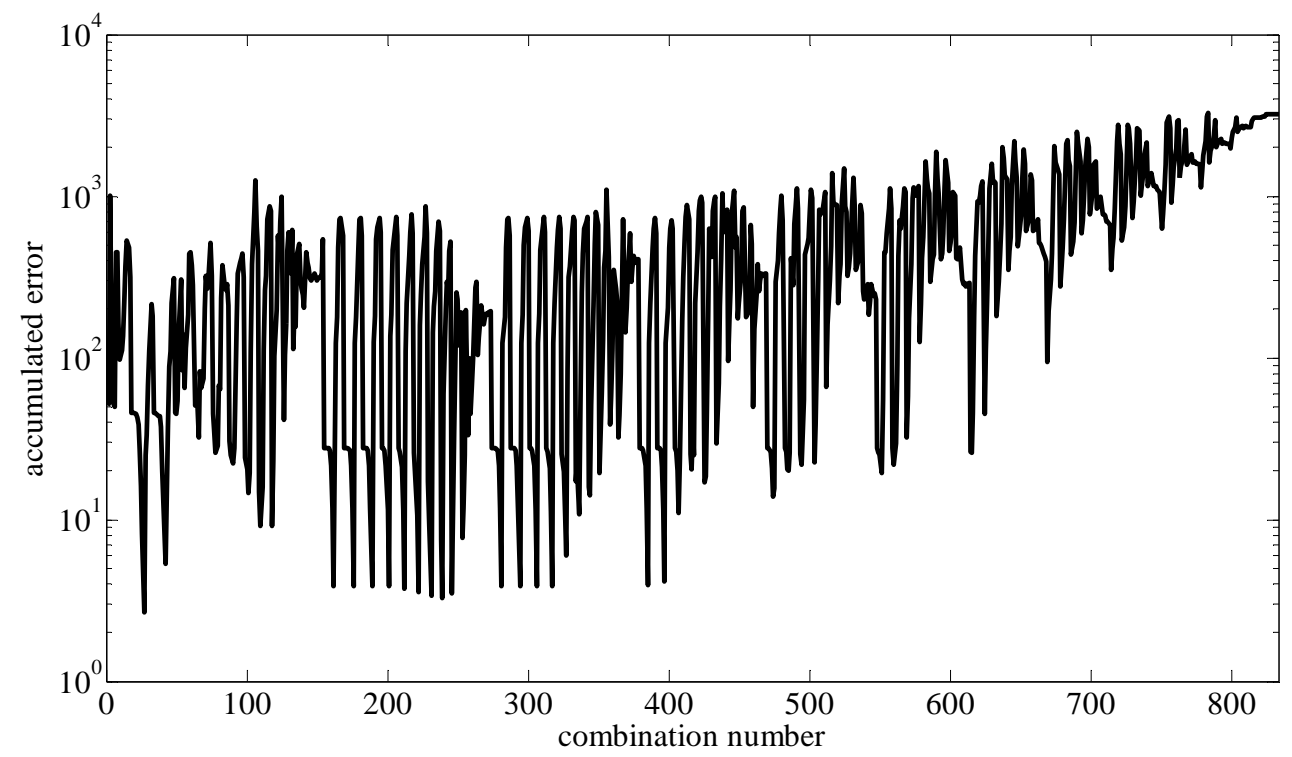

Figure 12. Accumulated error in frequency for each force combination.

As one can see, the absolute minimum corresponds to the right combination (no. 27), representing the forces applied at co-ordinates 1 and 11. So, the method located correctly the position of the forces. Note that, as there are two forces, a high number of combinations with small errors appear; observing the co-ordinates of those combinations, the best of them have in common the correct co-ordinates where the forces are applied and the others include coordinates physically close to them. Again, the force reconstruction is obtained using the measured displacements:

$$
\left\{\begin{array}{c}
\tilde{Y}_{3} \\
\tilde{Y}_{7} \\
\tilde{Y}_{13} \\
\tilde{Y}_{17}
\end{array}\right\}=\left[\begin{array}{cc}
H_{3,1} & H_{3,11} \\
H_{7,1} & H_{7,11} \\
H_{13,1} & H_{13,11} \\
H_{17,1} & H_{17,11}
\end{array}\right]\left\{\begin{array}{l}
F_{1} \\
F_{11}
\end{array}\right\} \Leftrightarrow\left\{\begin{array}{l}
F_{1} \\
F_{11}
\end{array}\right\}=\left[\begin{array}{ll}
H_{3,1} & H_{3,11} \\
H_{7,1} & H_{7,11} \\
H_{13,1} & H_{13,11} \\
H_{17,1} & H_{17,11}
\end{array}\right]^{+}\left\{\begin{array}{c}
\tilde{Y}_{3} \\
\tilde{Y}_{7} \\
\tilde{Y}_{13} \\
\tilde{Y}_{17}
\end{array}\right\}
$$

Figs. 13 and 14 present the reconstructed forces versus the measured ones. Again, one can state that the method is able to predict the applied force. 


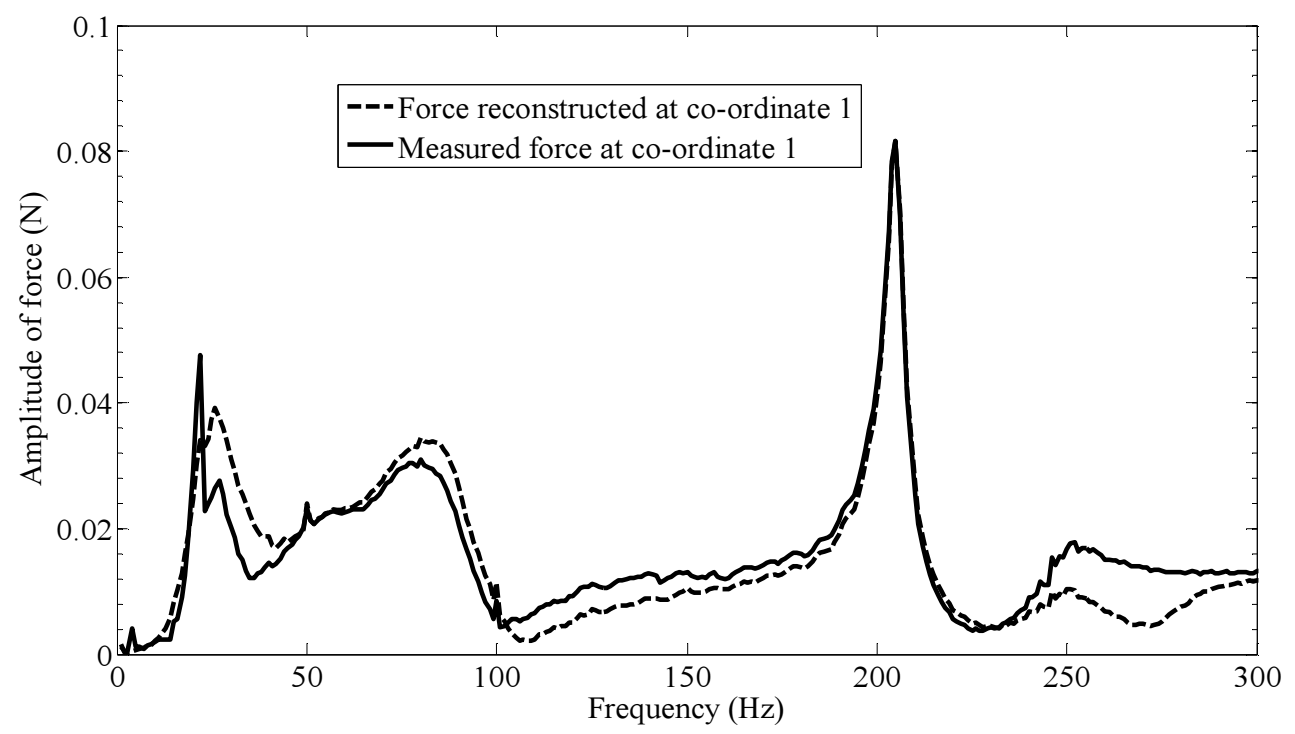

Figure 13. Comparison between the experimental and the reconstructed forces at co-ordinate 1

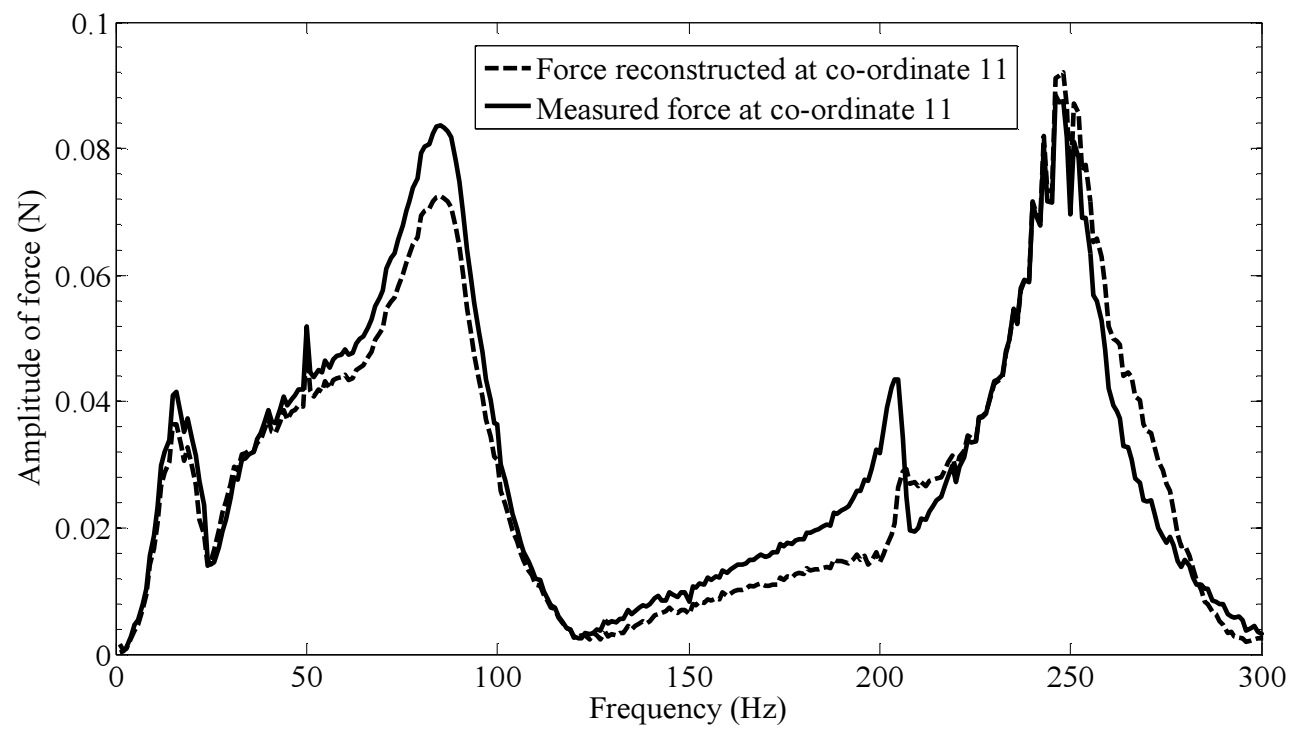

Figure 14. Comparison between the experimental and the reconstructed forces at co-ordinate11 


\subsection{Force reconstruction based on the transmissibility of forces}

The main objective of this section is the estimation of the existing forces (reactions or applied forces) in the structure using the MDOF concept of transmissibility of forces. Two types of problems involving estimation of forces are here considered:

1. Reaction forces estimation, with the objective of calculating a set of unknown reactions from a set of known applied loads, as expressed by equation (15);

2. Applied forces estimation, with the objective of calculating a set of applied forces from a set of known reactions, as expressed by equation (17).

The method to estimate the applied forces is limited by the number of reactions, as it is not possible to perform the needed pseudo-inverse if the number of applied forces is greater than the number of reactions. So, it is a required condition that $n_{K} \leq n_{U}$.

\subsubsection{Example 6 - Reaction forces estimation knowing the applied ones}

The first experimental reconstruction case was carried out with the configuration presented in Fig. 6 (simple supported beam). One has a single applied force at node 7 (set $K$ ) and two reactions at nodes 1 and $17(\operatorname{set} U)$.

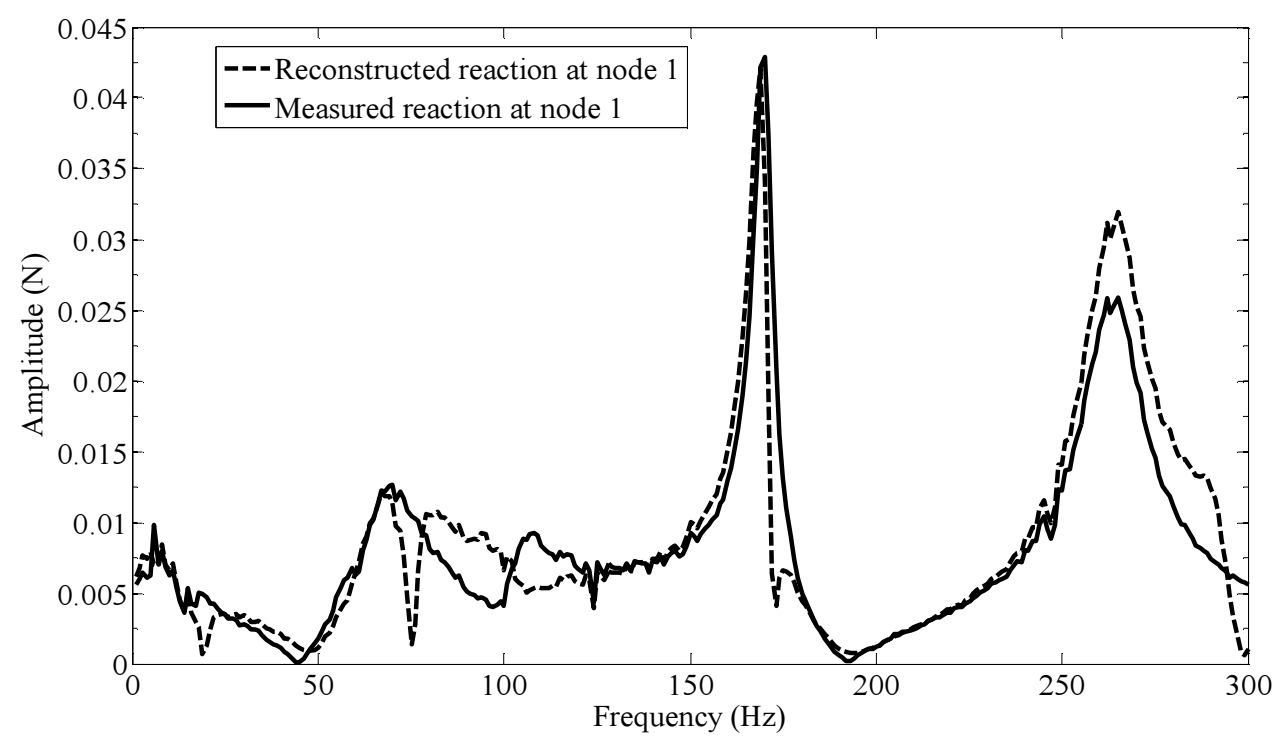

Figure 15. Comparison between the experimental and estimated force reaction $F_{1}$

In this case, with one applied force and two reactions, the transmissibility has a dimension of $2 x 1$ and can be obtained either from the receptance matrix or from the dynamic stiffness matrix, 
as proposed in this work. The two different formulations are equivalent and are very close to the experimental results.

As the objective is to estimate the reaction forces, one needs the numerical model for the transmissibility matrix and to know the experimental vector of applied forces, which in this case has only one component. The calculation of the reactions is then reduced to the following form:

$$
\left\{\begin{array}{l}
F_{1} \\
F_{17}
\end{array}\right\}=\left[\begin{array}{c}
T_{1,7} \\
T_{17,7}
\end{array}\right]\left\{F_{7}\right\}
$$

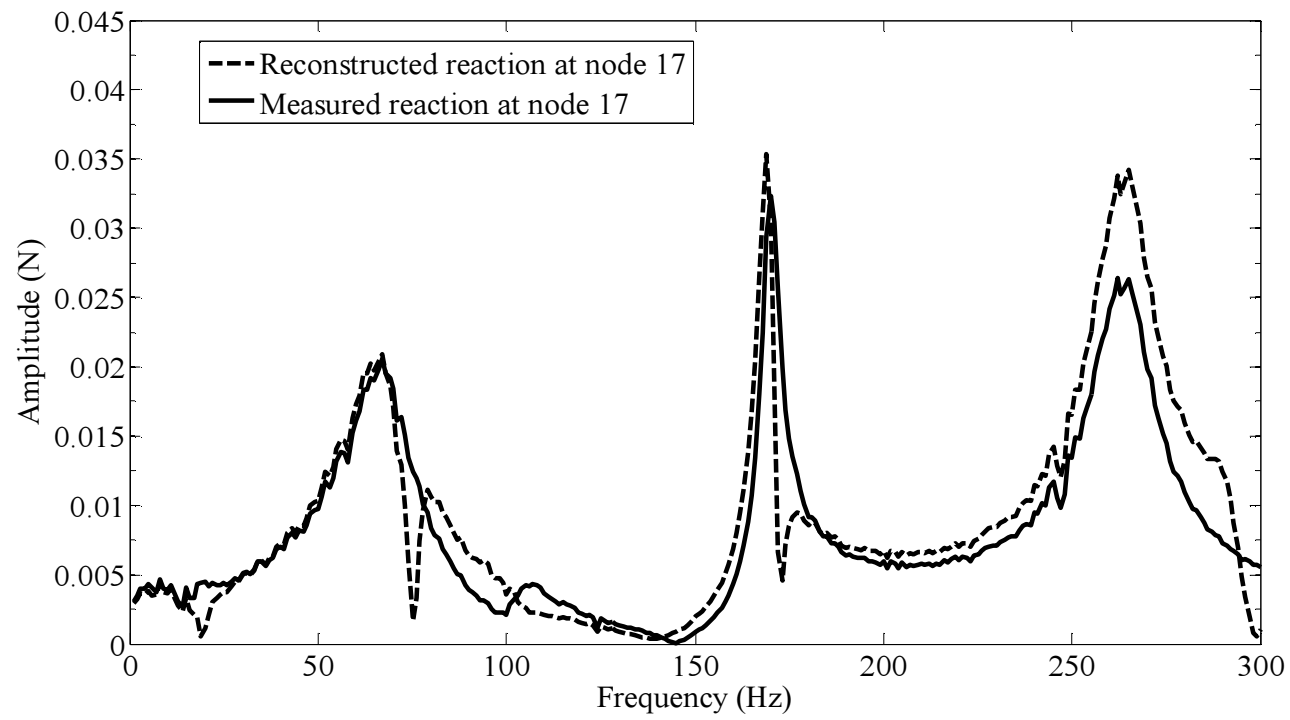

Figure 16. Comparison between the experimental and estimated force reaction $\mathrm{F}_{17}$

From Figs. 15 and 16, it is clear that the reconstructed reactions match reasonably well the experimentally measured ones. Better results may even be possible if a finer updating procedure on the FE model is achieved.

\subsubsection{Example 7 - Applied force reconstruction knowing the reaction forces}

For the reconstruction of the applied forces (the inverse problem), one needs to known the vector of the reaction forces $\left\{F_{U}\right\}$ and the inverse transmissibility matrix that can be obtained from the numerical model.

In this case the same configuration presented in Fig. 6 was used (simple supported beam), with one applied force at node 7 (set $K$ ) and two reaction forces at nodes 1 and 17 (set $U$ ). 
Knowing the reaction forces, the reconstruction of the applied force follows, in this case, the following expression:

$$
\left\{F_{7}\right\}=\left[\begin{array}{l}
T_{1,7} \\
T_{17,7}
\end{array}\right]^{+}\left\{\begin{array}{l}
F_{1} \\
F_{17}
\end{array}\right\}
$$

The reconstructed values are compared with the experimentally measured ones, in Fig. 17.

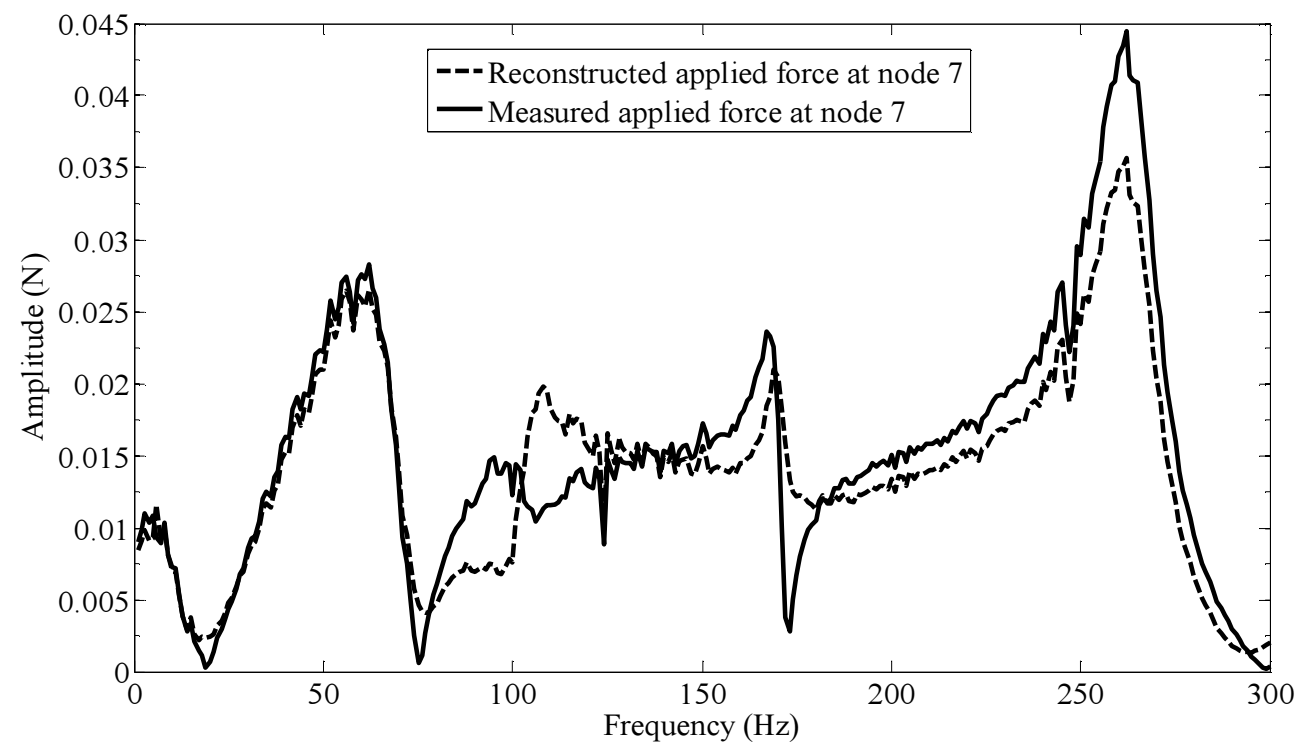

Figure 17. Comparison between the experimental and estimated applied load F7

In all the tested cases a good approach of the reconstructed forces was verified, as the values obtained by the direct and inverse problems are close enough to the experimentally measured ones.

\section{Conclusions}

In this work, the authors reviewed recent advances in the application of MDOF transmissibility-based methods for the identification of forces.

From these developments, one can draw the following main conclusions: 
i. it is possible to localize forces acting on a structure, based on the motion transmissibility matrix, comparing the expected responses with the ones measured along the structure;

ii. finding where the forces are applied corresponds to finding the transmissibility matrix related to the smallest error between the expected responses and measured ones along the frequency range;

iii. in all the examples the identification of the number of forces and their localization have been accomplished;

iv. the magnitude of the estimated forces exhibits a very good correlation with the measured ones for most of the frequency range.

\section{Acknowledgements}

The current investigation had the support of IDMEC/IST and FCT, under the project PTDC/ EME-PME/71488/2006.

\section{Author details}

N. M. M. Maia, Y. E. Lage and M. M. Neves

*Address all correspondence to: nmaia@dem.ist.utl.pt

IDMEC-IST, Technical University of Lisbon, Department Mechanical Engineering, Lisboa, Portugal

\section{References}

[1] Maia, N. M. M, Urgueira, A. P. V, \& Almeida, R. A. B. Whys and Wherefores of Transmissibility. In: Dr. Francisco Beltran-Carbajal (ed.) Vibration Analysis and Control- New Trends and Developments, InTech; (2011). http://www.intechopen.com/ books/vibration-analysis-and-control-new-trends-and-developments/whys-andwherefores-of-transmissibility., 187-216.

[2] Hillary, B. Indirect Measurement of Vibration Excitation Forces. PhD Thesis. Imperial College of Science, Technology and Medicine, Dynamics Section, London, UK; (1983).

[3] Stevens, K. K. Force Identification Problems- an overview. Proceedings of the (1987). SEM Spring Conference on Experimental Mechanics. Houston, TX, USA; 1987. 
[4] Mas, P, Sas, P, \& Wyckaert, K. Indirect force identification based on impedance matrix inversion: a study on statistical and deterministic accuracy. Proceedings of 19th International Seminar on Modal Analysis, Leuven. Belgium, (1994). , 1049-1065.

[5] Dobson, B. J, \& Rider, E. A review of the indirect calculation of excitation forces from measured structural response data. Proceedings of the Institution Mechanical Engineers, Part C, Journal of Mechanical Engineering Science,(1990). , 69-75.

[6] Ma, C. K, Chang, J. M, \& Lin, D. C. Input Forces Estimation of Beam Structures by an Inverse Method. Journal of Sound and Vibration, (2003). , 387-407.

[7] Ma, C. K, \& Ho, C. C. An Inverse Method for the Estimation of Input Forces Acting On Non-Linear Structural Systems. Journal of Sound and Vibration, (2004). , 953-971.

[8] Thite, A. N, \& Thompson, D. J. The quantification of structure-borne transmission paths by inverse methods. Part 1: improved singular value rejection methods. Journal of Sound and Vibration, (2003). , 411-431.

[9] Thite, A. N, \& Thompson, D. J. The quantification of structure-borne transmission paths by inverse methods. Part 2: use of regularization methods, Journal of Sound and Vibration, (2003). , 433-451.

[10] Choi, H, Thite, G, \& Thompson, A. N. D. J. A threshold for the use of Tikhonov regularization in inverse force determination. Applied Acoustics, (2006). , 700-719.

[11] Michaels, J. E, \& Pao, Y. H. The inverse source problem for an oblique force on an elastic plate. Journal of the Acoustic. Society of America, June (1985). , 2005-2011.

[12] Martin, M. T, \& Doyle, J. F. Impact force identification from wave propagation responses. International Journal of Impact Engineering, January (1996). , 65-77.

[13] Huang, C. H. An inverse non-linear force vibration problem of estimating the external forces in a damped system with time-dependent system parameters. Journal of Sound and Vibration, (2001). , 749-765.

[14] Tadeusz UhlThe inverse identification problem and its technical application, Arch Appl Mech, (2007). , 325-337.

[15] Zhang, Q, Allemang, R. J, \& Brown, D. L. Modal Filter: Concept and Applications, 8th International Modal Analysis Conference (IMAC VIII), Kissimmee, Florida, (1990). , 487-496.

[16] Kammer, D. C, \& Steltzner, A. D. Structural identification of Mir using inverse system dynamics and Mir/shuttle docking data, Journal of Vibration and Acoustics, (2001). , 230-237.

[17] Allen, M. S, \& Carne, T. G. Comparison of Inverse Structural Filter (ISF) and Sum of Weighted Accelerations (SWAT) Time Domain Force Identification Methods, Mechanical Systems and Signal Processing,(2008). , 1036-1054. 
[18] Paulo, P. A Time-Domain Methodology For Rotor Dynamics: Analysis and Force Identification. MSc thesis. Instituto Superior Técnico Lisbon; (2011).

[19] Vakakis, A. F. Dynamic Analysis of a Unidirectional Periodic Isolator, Consisting of Identical Masses and Intermediate Distributed Resilient Blocks. Journal of Sound andVibration, (1985). , 25-33.

[20] Vakakis, A. F, \& Paipetis, S. A. Transient Response of Unidirectional Vibration Isolatorswith Many Degrees of Freedom, Journal of Sound and Vibration, (1985). 0002-2460X., 99(4), 557-562.

[21] Vakakis, A. F, \& Paipetis, S. A. The Effect of a Viscously Damped Dynamic Absorber ona Linear Multi-Degree-of-Freedom System, Journal of Sound and Vibration, (1986). (1), 49-60.

[22] Liu, W. Structural Dynamic Analysis and Testing of Coupled Structures. PhD thesis. Imperial College London; (2000).

[23] Liu, W, \& Ewins, D. J. Transmissibility Properties of MDOF Systems, Proceedings of the16th International Operational Modal Analysis Conference (IMAC XVI), Santa Barbara, California, (1998). , 847-854.

[24] Varoto, P. S, \& Mcconnell, K. G. Single Point vs Multi Point AccelerationTransmissibility Concepts in Vibration Testing, Proceedings of the 12th International Modal Analysis Conference (IMAC XVI), Santa Barbara, California, USA,(1998). , 83-90.

[25] Ribeiro, A. M. R. On the Generalization of the Transmissibility Concept, Proceedings of the NATO/ASI Conference on Modal Analysis and Testing, Sesimbra, Portugal, (1998). , 757-764.

[26] Fontul, M, Ribeiro, A. M. R, Silva, J. M. M, \& Maia, N. M. M. Transmissibility Matrix in Harmonic and Random Processes, Shock and Vibration, (2004). , 563-571.

[27] Maia, N. M. M, Fontul, M, \& Ribeiro, A. M. R. Transmissibility of Forces in MultipleDegree-of-Freedom Systems, Proceedings of ISMA (2006). Noise and Vibration Engineering, Leuven, Belgium 2006.

[28] Rao, S. S. Mechanical Vibrations. Fourth International Edition. Prentice-Hall; (2004). 